\title{
Failure to replicate moral licensing and moral cleansing in an online experiment
}

\author{
Amanda Rotella \& Pat Barclay
}

Amanda Rotella

Department of Psychology

University of Waterloo

* Corresponding Author

a.rotella@kingston.ac.uk
Pat Barclay

Department of Psychology

University of Guelph

barclayp@uoguelph.ca

Notes:

1. This paper is published in Personality and Individual Differences:

Rotella, A., \& Barclay, P. (2020). Failure to replicate moral licensing and moral cleansing in an online experiment. Personality and Individual Differences, 161, 109967.

2. PI's current affiliation is Kingston University. Please contact Amanda Rotella at a.rotella@kingston.ac.uk 


\begin{abstract}
Moral licensing occurs when someone who initially behaved morally or cooperatively, later behaves less morally, as if they had a "license" to act badly. On the flipside, moral cleansing occurs when someone first behaves immorally, which prompts them to later behaves more morally. To-date, few studies have investigated individual differences in the moral licensing and cleansing effects. This paper bridges this gap by investigating how cooperative preferences, as measured by social value orientation (SVO), influence engagement in these effects. We hypothesized that prosocial participants would be less likely to license, but more likely to cleanse. Contrary to predictions, we did not replicate the moral licensing or moral cleansing effects, and cooperative preferences did not influence engagement in the effect. However, checks suggest that our manipulations were successful. We postulate that licensing and cleansing effects are unlikely to be elicited online.
\end{abstract}

Keywords: Moral licensing, moral balancing, moral cleansing, moral compensation, social value orientation 


\subsection{INTRODUCTION}

Moral licensing occurs when someone who initially behaved morally or cooperatively,

3 later behaves less morally or cooperatively, as if they had a "license" to act badly. This effect has

4 been reported in many domains, including cooperation (e.g., Conway \& Peetz, 2012),

5 environmentalism (e.g., Geng et al., 2016), and discrimination (e.g., Monin \& Miller, 2001).

6 Recent meta-analyses suggest that this is a small, but real effect (Blanken et al., 2015; Kuper \&

7 Bott, 2018). On the flipside, the moral cleansing effect happens when people who have behaved

8 immorally, subsequently behave more cooperatively as if they need "cleanse themselves of" their

9 bad deeds (Conway \& Peetz, 2012; Jordan et al., 2011). Moral cleansing has also been found

10 across different domains, including cooperation (Conway \& Peetz, 2012), and cheating (Jordan

11 et al., 2011).

12 Despite the vast literature on moral licensing and moral cleansing, with over 400 articles

13 discussing these topics, few papers have investigated individual differences in these effects (see

14 supplement). Moreover, studies have not yet tested whether differences in cooperativeness

15 influence susceptibility to engage in moral licensing or compensation.

16

We posit that the moral licensing and cleansing effects will be influenced by participants'

17 cooperativeness, where cooperators will be less likely to engage in moral licensing but more

18 likely to engage in moral cleansing. Recent theoretic and empirical work on the morality as

19 cooperation hypothesis suggest that morality is a collection of cultural and biological solutions

20 that solve recurrent problems of human cooperation (Curry et al., 2019). This suggests that

21 morality and cooperation are strongly overlapping constructs, and can be similarly

22 operationalized. Thus, we can predict a moral licensing effect for cooperative manipulations and 
23 dependent measures, in which moral licensing effects have been found (discussion in 24 supplement).

In this study, we characterize peoples' cooperativeness by their social value orientation

26 [SVO]), which is a points-based measure of how much a person values someone else, relative to

27 themselves. SVO has been associated with many real outcomes, including generosity in

28 economic games (Yamagishi et al., 2013), and sacrifice in real-life social relations (Van Lange et 29 al., 1997).

We hypothesize that people who prefer equal outcomes (i.e., prosocials) will cooperate whether or not they have acquired a 'license'. On the flipside, we posit that individuals who aim

32 to maximize their earnings, would use good behaviours to justify being uncooperative, and

33 therefore 'license'. Similarly, for moral cleansing, we posit that cooperators will be more likely to compensate (and cleanse) after recalling immoral behaviours, compared to individuals who

35 are more selfish. The purpose of this study is to investigate how individual differences in SVO influence susceptibility to moral licensing and cleansing effects.

\subsection{METHODS}

This experiment was pre-registered at osf.io/8bm5g; data, analysis scripts, and supplementary material are available at https://osf.io/f8byg/. See supplement for pre-screening 40 details.

\section{$41 \quad$ 1.2.1 Participants}

A total of 562 Amazon Mechanical Turk Workers from the United States were paid to 43 complete a "Short survey about past behaviour and decisions". Based on our pre-registered 44 exclusion criteria, 44 participants were excluded (see supplement). The final sample had 519 45 participants $($ Mage $=37.32$, SDage $=12.29 ; 57.2 \%$ female, $41.4 \%$ male, $1.3 \%$ other $)$, with 164 
participants in the cooperative condition, 163 in the neutral condition, and 192 in the uncooperative condition. For SVO, 319 participants were categorized as prosocial and 200 as egoists.

\subsubsection{Survey}

Participants were randomly assigned to recall one of three types of recent behaviors, which occurred in the last month: cooperative/moral (hereafter cooperative), neutral, or uncooperative/immoral (hereafter uncooperative). Then, participants described that event. For example, in the cooperative condition the prompt was: "Please recall a time when you acted in such a way that you felt virtuous or honorable. Perhaps you were loyal to a friend, were generous when you could have been selfish, were kind to someone for no particular reason, or example item). Scores were calculated and converted to a number on a Cartesian plane, where

\footnotetext{
${ }^{1}$ Donations were made to these charities on participants' behalves at the completion of this study.
} 
68 (see Murphy et al., 2011). There are three SVO 'types': prosocials who prefer to maximize joint 69 gain (i.e. choose to distribute points equally), egoists prefer to maximize their own outcome (i.e. 70 typically choose the maximum amount and disregarding the amount for the other), and 71 competitors who prefer to maximize the difference between themselves and the other (i.e.

72 typically choose the option that has the greatest difference between themselves and the other).

73 However, data were analyzed as a continuous variable.

74 The SVO slider was presented after our target measures to minimize and after

75 demographics to minimize carry-over effects. To ensure that participants adequately completed 76 the writing task, independent raters assessed the cooperativeness and relevance of the responses 77 (see Supplement).

78 $\quad$ 1.2.3 Analyses

79 We used ANOVAs in R version 3.6.0 (R Core Team, 2013) and interpreted them using 80 the New Statistics (Cumming, 2012). We also provide null hypothesis significance tests, 81 although these were not pre-registered (see Supplement; additional analyses and descriptives in 82 supplement).

\section{$84 \quad$ 1.3.1 Manipulation Checks}

85 Blinded raters scored the cooperativeness of participant responses. There was a large

86 effect of condition, $F(2,516)=1207, p<.001$, Cohen's $f=2.16$, where the cooperative

87 responses were rated as more cooperative than neutral responses $(d=3.95)$ and uncooperative

88 responses, $d=4.69$. Neutral responses were rated more cooperatively than uncooperative

89 responses, $d=2.04$. 
Participants also responded to the question "When recalling and describing a time where

91 you felt [insert condition-specific statement], how cooperative did you feel??. There was a large

92 effect of condition $(F(2,515)=112.1, p<.001$, Cohen's $f=0.66)$, with people feeling more

93 cooperative in the cooperative condition than the neutral $(d=1.27)$ and uncooperative $(d=1.51)$

94 conditions. They felt less cooperative in the uncooperative condition compared to the neutral

95 condition $(d=0.54)$. Results from both analyses suggest that the manipulation was successful

96 (Figure 1; detailed analyses in supplement).

97
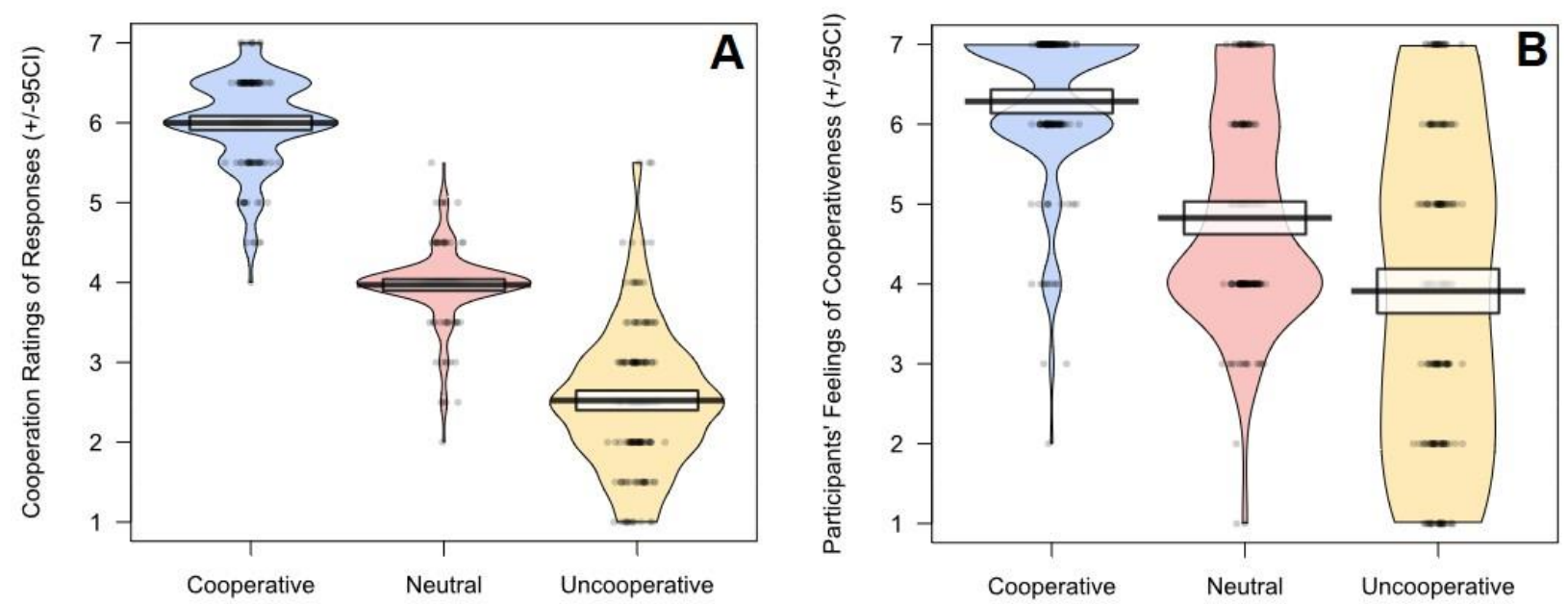

98

Figure 1. Manipulation check: violin plots of (a) raters' cooperativeness ratings of participant responses in the manipulation by experimental condition, and (b) participants' cooperative 101 feelings following the manipulation by condition. Ratings were completed on 7-point Likert

102 Scales. Means are indicated by the black line, and the white boxes are $95 \%$ confidence intervals.

103 Dots represent data points (with jitter) and the colored areas represent the response distributions.

\subsubsection{Main Analyses}


There was a small and marginal effect of condition on donation, $F(2,508)=2.93, p=$ .054 , Cohen's $f=.11$. Contrary to predictions, participants in the cooperative condition $(M=$ $10.19, S D=10.34,95 \mathrm{CI}[8.62,11.81])$ donated significantly more than those in the neutral condition $(M=7.65, S D=9.34,95 \mathrm{CI}[6.12,9.00] ; t(321.77)=2.43, p=.015$, Cohen's $d=0.27$, $95 \mathrm{CI}[0.05,0.49]$, and marginally more than the uncooperative condition $(M=8.36, S D=9.55$, 95CI[7.00, 9.72]), $t(334.32)=1.75, p=.082$, Cohen's $d=0.19,95 \mathrm{CI}[-0.02,0.40]$. Both were small effects. Donations in the neutral and uncooperative conditions did not differ, $t(345.53)=$ $0.79, p=.428$, Cohen's $d=0.08,95 \mathrm{CI}[-0.12,0.29]$. These results showed a (small) consistency effect in the cooperative condition (i.e., opposite direction of predicted effect), and we did not find either moral licensing cleansing effects.

We computed a factorial ANOVA to determine the influence of SVO and licensing condition on amount donated to charity. SVO had a large effect on donation amount, $F(1,512)=$ 99.05, $p<.001$, Cohen's $f=0.44$, where participants who were more prosocial (i.e., higher SVO scores) donated more $(r=.40,95 \mathrm{CI}[.33, .47], p<.001)$. In this analysis, condition did not influence amounts donated, $F(2,512)=1.34, p=.263$, Cohen's $f=0.07$, and contrary to our predictions SVO and condition did not interact, $F(2,512)=0.22, p=.789$, Cohen's $f=0.03$. See Figure 2. Qualitatively similar results were obtained when using the proportion of participants who gave as a DV, and when we excluded all participants who did not give anything (see supplement). 


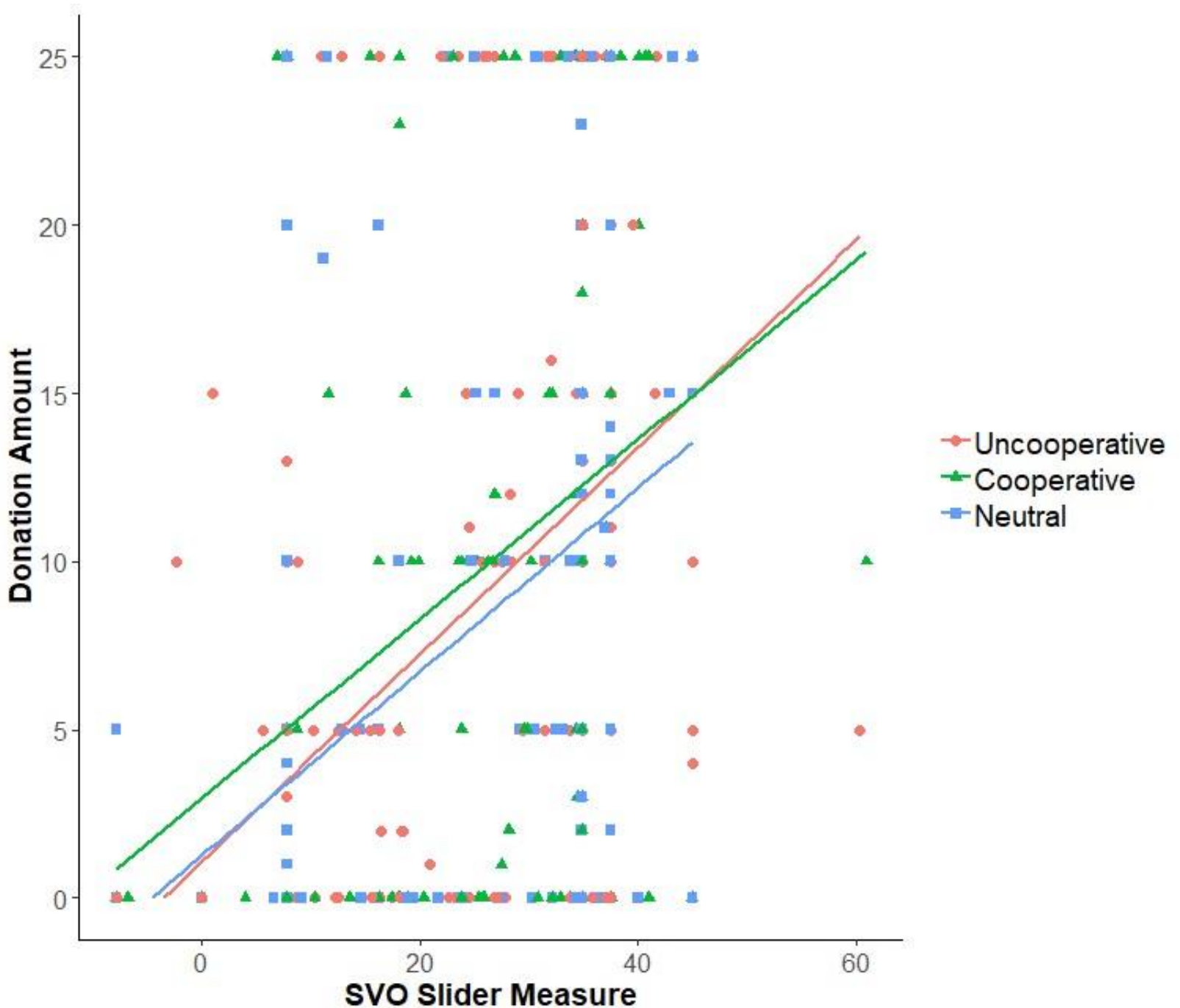

Figure 2. Amount donated to charity (\$) according to SVO score and experimental condition.

127 Higher SVO scores indicate greater prosociality.

131 good behaviour had a small consistency effect (i.e., an increase in donations), and recalling a past

132 bad behaviour did not influence donation amounts. This finding is inconsistent with the moral

133 licensing/cleansing literature, but consistent with moral consistency literature (Balliet, Parks, \&

134 Joireman, 2009). Our manipulation was highly successful: participants reported feeling more 
cooperative after completing the cooperative/moral manipulation than the neutral manipulation, and less cooperative after the uncooperative/immoral manipulation.

Similarly, recent exact and conceptual replications have failed to find moral licensing and cleansing effects (Blanken, van de Ven, Zeelenberg, \& Meijers, 2014; Urban, Bahník, \& Kohlová, 2019), suggesting that licensing and cleansing effects are not always be elicited. Conway and Peetz' (2012) studies used similar methodology, however they found licensing/cleansing effects. These differences may be due to: (i) spurious effects (our sample size was three times larger), (ii) methodological differences (no control, different dependent measures), or (iii) Mturk workers are habituated to licensing and cleansing primes. Future research should replicate this effect online in naïve populations.

A new meta-analysis suggests that the moral licensing effect may be calibrated through reputation, where people will only 'license' if they have established to others that they are a good person. When no one is watching, participants do not establish a license (Rotella et al., 2019). Moreover, the ambiguity of the DV will influence when people license and when they do not, such that people are more likely to license with ambiguous dependent measures (Rotella et al., 2019). These factors help explain why we did not find a moral licensing effect - there were no reputation-based cues (e.g., observation) in this online study, and the dependent measure was unambiguous. Given that moral cleansing is the flipside of moral licensing, it may also be affected by reputational cues.

SVO influences responses to reputational cues. In the absence of reputational cues, proselfs (i.e., egoists/competitors) are less cooperative than prosocials, but they are equally cooperative when reputational cues are present (Simpson \& Willer, 2008). Given that proselfs 
157 are more strategic in their cooperation, we posit that individual differences in moral licensing and 158 cleansing effects will only be elicited when there are reputation-based reasons to license.

159 Nevertheless, this study adds to the literature in several important ways. Firstly, in the 160 absence of reputational cues (i.e., online, no observation), recalling a past good or bad behaviour 161 does not appear to influence subsequent cooperative behaviours. Thus, we failed to replicate 162 Conway and Peetz (2012). Secondly, we nevertheless validated the licensing manipulation 163 adapted from Conway and Peetz (2012): participants completed the task as requested, which 164 elicited more (less) cooperative feelings after reporting a good (bad) deed, but those feelings do 165 not cause moral licensing (cleansing). Thirdly, SVO does not influence moral licensing and 166 cleansing, at least not in this study; future studies should retest this using different methodology 167 (e.g., immoral dependent measure). Lastly, SVO prosocials donated more to charity, which 168 provides further validation that SVO predicts real-world behaviours. We failed to replicate moral licensing and cleansing effects. Recalling past good/bad

171 behaviours did not influence donations to charity, and this did not differ by cooperative

172 preferences, possibly because of a lack of overall effect. Given that our manipulations were 173 successful and there is recent evidence that moral licensing is calibrated through reputation174 based mechanisms rather than self-image (Rotella et al., 2019), we posit that moral licensing and 175 cleansing effects are unlikely to be elicited online. 


\subsection{REFERENCES}

Balliet, D., Parks, C., \& Joireman, J. (2009). Social value orientation and cooperation in social dilemmas: A meta-analysis. Group Processes \& Intergroup Relations, 12(4), 533-547.

Blanken, I., van de Ven, N., \& Zeelenberg, M. (2015). A meta-analytic review of moral licensing. Personality and Social Psychology Bulletin, 41(4), 540-558.

Blanken, I., van de Ven, N., Zeelenberg, M., \& Meijers, M. H. (2014). Three attempts to replicate the moral licensing effect. Social Psychology, 45(3), 232.

Conway, P., \& Peetz, J. (2012). When does feeling moral actually make you a better person? Conceptual abstraction moderates whether past moral deeds motivate consistency or compensatory behavior. Personality and Social Psychology Bulletin, 38(7), 907-919.

Cumming, G. (2012). Understanding the New Statistics: Effect Sizes. Confidence Intervals, and Meta-Analysis.

Curry, O. S., Mullins, D. A., \& Whitehouse, H. (2019). Is it good to cooperate? Testing the Theory of Morality-as-Cooperation in 60 Societies, Current Anthropology, 60(1), 47-

69.Geng, L., Cheng, X., Tang, Z., Zhou, K., \& Ye, L. (2016). Can previous pro-environmental behaviours influence subsequent environmental behaviours? The licensing effect of proenvironmental behaviours. Journal of Pacific Rim Psychology, 10.

Jordan, J., Mullen, E., \& Murnighan, J. K. (2011). Striving for the moral self: The effects of recalling past moral actions on future moral behavior. Personality and Social Psychology Bulletin, 37(5), 701-713.

Kuper, N., \& Bott, A. (2019). Has the evidence for moral licensing been inflated by publication bias?. Meta-Psychology, 3 .

R Core Team (2013). R: A language and environment for statistical computing. 
Rotella, A., Jung, J., Chinn, C., \& Barclay, P. (2019). Observation and moral ambiguity matter: A meta-analysis on moral licensing. Manuscript in preparation.

Monin, B., \& Miller, D. T. (2001). Moral credentials and the expression of prejudice. Journal of Personality and Social Psychology, 81(1), 33.

Murphy, R. O., Ackermann, K. A., \& Handgraaf, M. (2011). Measuring social value orientation. Judgment and Decision Making, 6(8), 771-781.

Simpson, B., \& Willer, R. (2008). Altruism and indirect reciprocity: The interaction of person and situation in prosocial behavior. Social Psychology Quarterly, 71(1), 37-52.

Urban, J., Bahník, Š., \& Kohlová, M. B. (2019). Green consumption does not make people cheat: Three attempts to replicate moral licensing effect due to pro-environmental behavior. Journal of Environmental Psychology, 63, 139-147.

Van Lange, P. A., De Bruin, E., Otten, W., \& Joireman, J. A. (1997). Development of prosocial, individualistic, and competitive orientations: theory and preliminary evidence. Journal of personality and social psychology, 73(4), 733.

Yamagishi, T., Mifune, N., Li, Y., Shinada, M., Hashimoto, H., Horita, Y., ... \& Takagishi, H. (2013). Is behavioral pro-sociality game-specific? Pro-social preference and expectations of pro-sociality. Organizational Behavior and Human Decision Processes, 120(2), 260271. 